\title{
Aronia-Zubereitungen wirken günstig auf den Lipidstoffwechsel
}

Hierzulande neuerdings als exotisches „Superfood“ gehandelt, gilt die Apfelbeere Aronia melanocarpa aus der Familie der Rosaceae in ihrer Heimat Nordamerika schon seit Jahrhunderten als traditionelle Heilpflanze bei Erkältungskrankheiten. Untersuchungen ihrer Inhaltsstoffe zeigen einen hohen Gehalt an phenolischen Verbindungen, Anthocyanen, Ascorbinsäure und Antioxidanzien.

Rahmani J et al. The effect of Aronia consumption on lipid profile, blood pressure, and biomarkers of inflammation: A systematic review and meta-analysis of randomized controlled trials. Phytother Res 2019; 33: 1981-1990

Hierzulande neuerdings als exotisches „Superfood“ gehandelt, gilt die Apfelbeere Aronia melanocarpa aus der Familie der Rosaceae in ihrer Heimat Nordamerika schon seit Jahrhunderten als traditionelle Heilpflanze bei Erkältungskrankheiten. Untersuchungen ihrer Inhaltsstoffe zeigen einen hohen Gehalt an phenolischen Verbindungen, Anthocyanen, Ascorbinsäure und Antioxidanzien.

Insbesondere die Polyphenole der Apfelbeere sind wissenschaftlich von Interesse, da sie sich in Tierversuchen und klinischen Studien beim Menschen als Sekundärmetaboliten an sich und je nach Verbindung individuell als antiproliferativ, antimikrobiell, antiinflammatorisch und antidiabetisch wirksam erwiesen.

Eine Supplementation mit Extrakten oder Saft aus Aronia-Beeren zeigte in klinischen Studien bei Rauchern, bei Kindern und Erwachsenen mit metabolischem Syndrom einen günstigen Einfluss auf den Lipidstoffwechsel und einzelne kardiovaskuläre Biomarker. Eine umfassende Metaanalyse einer internationalen Arbeitsgruppe sollte nun die bisherige klinische Evidenz einer Aronia-Supplementation in Bezug auf die Lipidwerte, den Blutdruck und Entzündungsmarker zusammenfassen.

Das Studienteam wertete nach einer umfassenden Literaturrecherche in den medizinischen Datenbanken PubMed/ MEDLINE, Scopus und Cochrane letztlich 6 Publikationen mit insgesamt 7 Studien aus, die nach Anlegen der vordefinierten Ein- und Ausschlusskriterien von ursprünglich 195 Studien zu Aronia übrig geblieben waren. Sie basierten alle auf einem randomisiert-kontrollierten Studiendesign bei erwachsenen Teilnehmern. Als Endpunkte sollten die Studien die Veränderung im Lipidprofil (Cholesterin, LDLund HDL-Werte, Triglyceride), den systolischen und diastolischen Blutdruck und die Entzündungsmarker C-reaktives Protein, Interleukin-6 und Tumor-Nekrose-Faktor $\alpha$ enthalten haben.

Im Mittel dauerten die Studien 16 Wochen (4-24 Wochen) mit einer Teilnehmerzahl zwischen 15 und 77 und einem Durchschnittsalter von 36 Jahren. Die Teilnehmer hatten durchschnittlich $228 \mathrm{ml}$ bzw. mg/Tag (100-500 ml bzw. $\mathrm{mg} / \mathrm{Tag}$ ) Aronia-Produkte eingenommen.

Nach der statistischen Auswertung konnten die Autoren belegen, dass eine Aronia-Supplementation den HDLWert und den diastolischen (nicht den systolischen) Blutdruck signifikant erhöhte. Letzterer Befund beruht allerdings auf nur zwei Studien mit zusammen 86 Teilnehmern. In einer Subgruppenanalyse hinsichtlich der Dauer der Einnahme senkte die AroniaEinnahme auch deutlich den LDL-Spiegel und das Gesamtcholesterin bei den Studien mit einer eher kürzeren
Studiendauer (unter 10 Wochen). Auch die Triglycerid-Werte nahmen in einer Dosis-Wirkungs-Analyse mit steigender Extrakt-Einnahme bis $300 \mathrm{mg} /$ Tag signifikant ab. Auf den systolischen Blutdruck und die Entzündungsmarker wirkten sich die Zubereitungen hingegen nicht wesentlich aus. Es machte auch keinen Unterschied, ob die Probanden die Früchte von Aronia melanocarpa als Saft oder als getrocknetes Nahrungsergänzungsmittel eingenommen hatten, da laut Autoren auch die verwendeten Extrakte alle relevanten bioaktiven Inhaltsstoffe enthielten.

\section{FAZIT}

In der Metaanalyse über 7 randomisiert-kontrollierte Studien zeigte sich, dass der Konsum von Zubereitungen aus Aronia melanocarpa den Lipidstoffwechsel günstig beeinflusst, indem die HDL-Werte signifikant ansteigen. Bei kürzerer Einnahme von unter 10 Wochen verringerte die Supplementation auch den LDL-Wert sowie das Gesamtcholesterin. Aufgrund der traditionellen Erfahrung mit dieser Pflanze sehen die Autoren das Potenzial des Aronia-Extrakts in einer therapiebegleitenden Supplementation insbesondere da sie im Gegensatz zu Lipidsenkern keine relevanten Sicherheitsrisiken aufweist. So könnte z. B. beim metabolischen Syndrom eine ergänzende Einnahme von AroniaExtrakt die Dosis an synthetischen Medikamenten verringern und damit auch deren potenzielle Nebenwirkungen abschwächen. Auf die isolierte Erhöhung des diastolischen Blutdrucks gehen die Autoren nicht ein.

Ulrike Andres, Ginsheim 